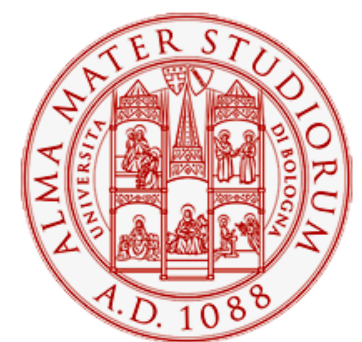

Alma Mater Studiorum - Università di Bologna DEPARTMENT OF ECONOMICS

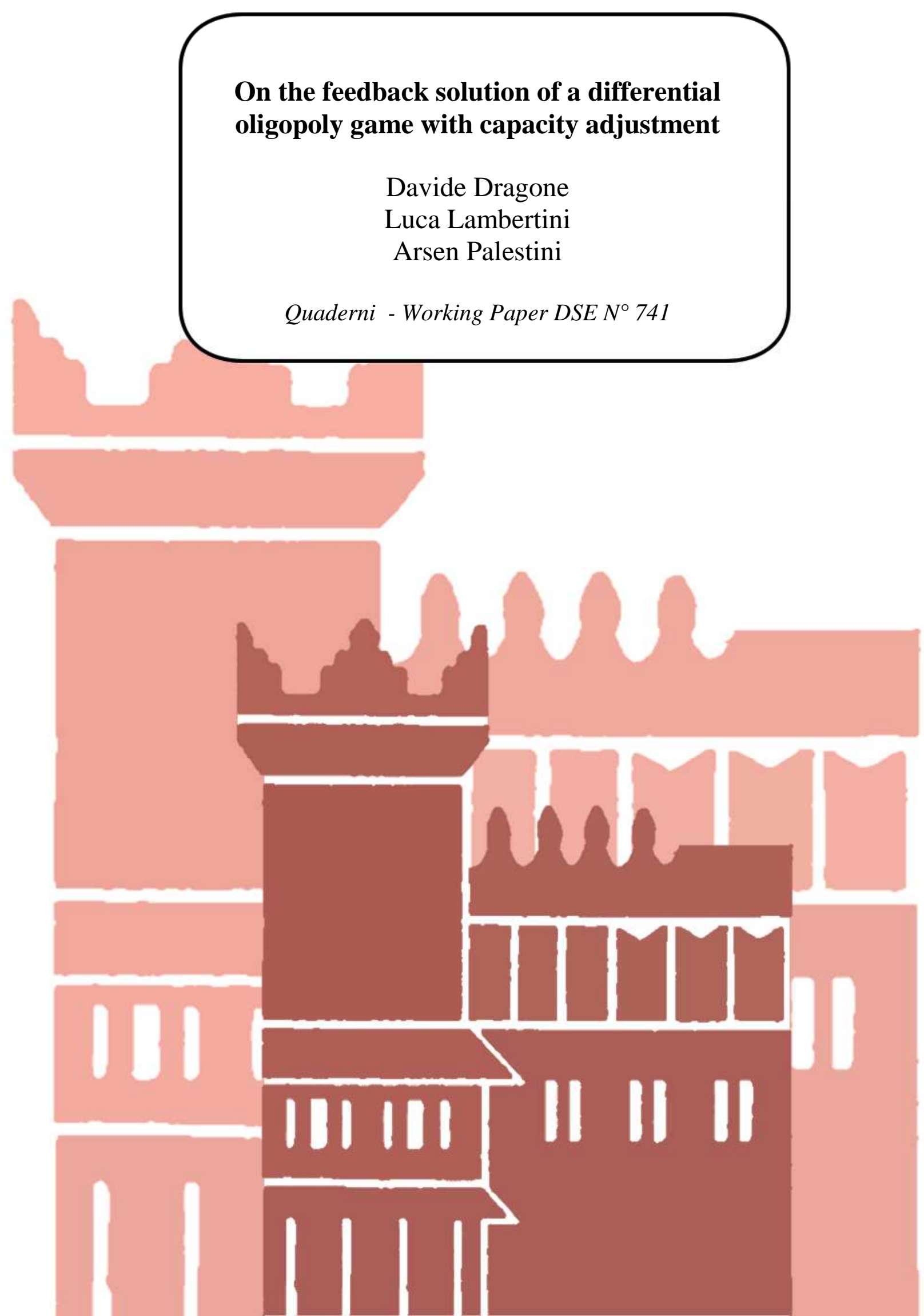




\title{
On the feedback solution of a differential oligopoly game with capacity adjustment ${ }^{1}$
}

\author{
Davide Dragone, ${ }^{*}$ Luca Lambertini* ${ }^{*}$ and Arsen Palestini ${ }^{\#}$ \\ * Department of Economics, University of Bologna \\ Strada Maggiore 45, 40125 Bologna, Italy \\ davide.dragone@unibo.it; luca.lambertini@unibo.it \\ $\S$ ENCORE, University of Amsterdam \\ Roetersstraat 11, WB1018 Amsterdam, The Netherlands \\ \# MEMOTEF, University of Roma "La Sapienza"
}

via del Castro Laurenziano 9, 00161 Roma, Italy; arsen.palestini@uniroma1.it

March 14, 2011

\footnotetext{
${ }^{1}$ We thank George Leitmann, Pierre Bernhard, Georges Zaccour and the audience at ISDG 2010 (Banff) and EARIE 2010 (Istanbul) for helpful comments and discussion. The usual disclaimer applies.
} 


\begin{abstract}
We propose a simple method for characterising analytically the feedback solution of oligopoly games with capital accumulation à la Solow-Swan. As a result, it becomes possible to contrast the feedback equilibrium against the corresponding one generated by open-loop information. Our method accomodates extensions of the stripped down oligopoly model in several directions. As an example, we expand the setup to include environmental effects and Pigouvian taxation.
\end{abstract}

JEL Classification: C73, L13, Q55

Keywords: capacity, differential game, feedback equilibrium, HamiltonJacobi-Bellman equation 


\section{Introduction}

The Solow-Swan model of economic growth with capital accumulation (Solow, 1956, Swan, 1956) is, together with its general equilibrium counterpart (Ramsey, 1928), a cornerstone of modern macroeconomics. Conversely, its corresponding interpretation as a strategic model in the field of industrial organization has received comparatively scanty attention. Yet, the availability of a fully analytical solution of a strategic model describing the costly accumulation of firms' productive capacity would certainly be desirable, as it would enable us to perform policy analyses in several relevant directions, such as regulation and taxation, international trade and FDI, the environmental implications of production and the exploitation of natural resources.

To the best of our knowledge, the only contributions to oligopoly theory examining setups where investment is reversible but capacity is subject to adjustment $\operatorname{costs}^{1}$ - as in the Solow-Swan model - are those of Fershtman and Muller (1984) and Reynolds (1987, 1991), while Cellini and Lambertini $(1998,2008)$ characterise the open-loop and (degenerate) feedback equilibria of an oligopoly à la Ramsey, where capacity accumulation is not explicitly costly but entails consumption (or sales) postponement. ${ }^{2}$

Here we propose a simpler (and more general) approach to the solution of the linear-quadratic differential oligopoly game à la Solow-Swan originally investigated by Reynolds $(1987,1991)$. His solution method involves the use of trigonometric functions as well as some numerical calculations. This ad-

\footnotetext{
${ }^{1}$ A similar approach, whereby adjusting output levels is costly, is adopted by Driskill and McCafferty (1989). The analysis of conjectural variations in a feedback oligopoly game with adjustment costs is in Dockner (1992). For a thorough discussion of oligopoly applications of differential game theory, see Jun and Vives (2004). A comprehensive overview of this literature is in Long (2010).

${ }^{2}$ Applications of these models to trade theory and policy can be found in Calzolari and Lambertini $(2006,2007)$.
} 
mittedly entails that the applicability of the model is hindered. Our method, instead, relies on some reasonable symmetry assumptions that yield a fully analytical solution of the Bellman equation of the representative firm.

This feedback solution is contrasted with the open-loop equilibrium of the same game, in order to investigate the consequences of increasing the amount of information used by firms on the limit properties of the model. This exercise allows us to single out a few interesting results. In particular, the maximum number of firms that can survive at the long run equilibrium is infinitely large under open-loop information while it is finite under feedback information. This fact is due to the higher degree of strategic interaction characterising the feedback game as compared to the open-loop one. The larger investment levels (i.e., higher costs) generated by the incentive to preempt rivals when feedback effects are operating, poses an upper bound to the population of firms. This, intuitively, implies that the long run industry structure at the feedback equilibrium is too concentrated as compared to what would be socially efficient.

To illustrate the potential applications of our solution method, we lay out an extension of the model allowing for the presence of a negative environmental externality associated with production, with firms being induced to carry out green R\&D efforts via the introduction of Pigouvian taxation.

The remainder of the paper is structured as follows. The model is laid out in section 2. Section 3 illustrates the open-loop Nash solution. The feedback equilibrium is investigated in section 4 . The analysis of the oligopoly with environmental externality is in section 5. Concluding remarks and suggestions for further applications are in section 6 . 


\section{The basic setup}

We examine a differential oligopoly game in continuous time $t \in[0, \infty)$, in which $N$ firms invest à la Solow-Swan to create productive capacity. Firm $i$ 's state and control variables are the capacity endowment $k_{i}(t)$ and the instantaneous investment $I_{i}(t)$, respectively, and the kinematic equation of capacity is

$$
\dot{k}_{i}(t)=I_{i}(t)-\delta k_{i}(t)
$$

where $\delta \geq 0$ is the constant decay rate of capacity, symmetric across all firms.

Firms operate at full capacity at every instant, and sell a homogeneous good. The inverse market demand function is linear:

$$
p(t)=A-\sum_{j=1}^{N} k_{j}(t) ; A>0 .
$$

Investment involves a convex instantaneous cost $\Gamma_{i}(t)=q I_{i}(t)+c I_{i}^{2}(t) / 2$. To guarantee the positivity of outputs and profits, we pose $A>q(\delta+\rho)$. The marginal cost associated with the production of the consumption good is constant, symmetric across firms and normalised to zero for the sake of simplicity. Hence, the instantaneous profit function of firm $i$ is:

$$
\pi_{i}(t)=\left[A-\sum_{j=1}^{N} k_{j}(t)\right] k_{i}(t)-q I_{i}(t)-\frac{c I_{i}^{2}(t)}{2}
$$

and firm $i$ chooses $I_{i}(t) \geq 0$ so as to maximise the discounted profit flow

$$
\Pi_{i}(t)=\int_{0}^{\infty} \pi_{i}(t) e^{-\rho t} d t
$$

s.t. the set of dynamic constraints $(2.1)$ and initial conditions $k_{i}(0)=k_{i 0}>$ 0 . The discount rate $\rho>0$ is constant and common to all firms. 
In the original Reynolds' model the state space is a compact set in the positive quadrant in which the net revenue is increasing, whereas each control variable is real. We intend to modify such hypotheses and replace them with the requirement that both the state space and the control space coincide with the positive orthant $\mathbb{R}_{+}^{N}$.

\section{The open-loop Nash equilibrium}

Under open-loop information, firm $i$ has to choose its instantaneous investment $I_{i}(t)$ so as to maximise the Hamiltonian function:

$$
\mathcal{H}_{i}(t)=\pi_{i}(t)+\lambda_{i i}(t) \dot{k}_{i}(t)+\sum_{j \neq i} \lambda_{i j}(t) \dot{k}_{j}(t)
$$

where $\lambda_{i j}(t)$ is the costate variable associated with the state $k_{j}(t)$, under the set of dynamic constraints given by the state equations (2.1) and initial conditions $k_{i}(0)=k_{i 0}>0$.

The necessary conditions are:

$$
\begin{gathered}
\frac{\partial \mathcal{H}_{i}(t)}{\partial I_{i}(t)}=-c I_{i}(t)-q+\lambda_{i i}(t)=0 \\
-\frac{\partial \mathcal{H}_{i}(t)}{\partial k_{i}(t)}=\dot{\lambda}_{i i}(t)-\rho \lambda_{i i}(t) \Leftrightarrow \\
\dot{\lambda}_{i i}(t)=\lambda_{i i}(t)(\delta+\rho)-A+2 k_{i}(t)+(N-1) k_{j}(t) \\
-\frac{\partial \mathcal{H}_{i}(t)}{\partial k_{j}(t)}=\dot{\lambda}_{i j}(t)-\rho \lambda_{i j}(t)
\end{gathered}
$$

where (3.4) can be disregarded as the rivals' capacities do not enter (3.2-3.3). 
Using (3.2-3.3) and imposing the symmetry conditions $I_{i}(t)=I_{j}(t)=$ $I(t) ; k_{i}(t)=k_{j}(t)=k(t)$ for all $i, j$, we obtain the following control dynamics: ${ }^{3}$

$$
\dot{I}=\frac{(\delta+\rho)(c I+q)-A+k(N+1)}{c}
$$

whereby the steady state equilibrium point of the open-loop Nash game is

$$
k_{O L}=\frac{A-q(\delta+\rho)}{N+1+c \delta(\delta+\rho)} ; I_{O L}=\delta k_{O L} .
$$

The following can be shown to hold: ${ }^{4}$

Proposition 3.1. The steady state $\left(k_{O L}, I_{O L}\right)$ is a saddle point equilibrium.

The resulting steady state profits are

$$
\pi_{O L}=\frac{\left[(A-q \delta)\left(2+c \delta^{2}\right)+\left(2 A c \delta+q\left(2 N-c \delta^{2}\right)\right) \rho\right][A-q(\delta+\rho)]}{2[N+1+c \delta(\delta+\rho)]^{2}}>0 .
$$

The equilibrium level of social welfare is:

$$
S W_{O L}=N \pi_{O L}+C S_{O L}=\frac{N[A-q(\delta+\rho)] \Gamma}{2[N+1+c \delta(\delta+\rho)]^{2}},
$$

where

$$
\Gamma=A[N+2+c \delta(\delta+2 \rho)]-q\left[c \delta^{2}(\delta+\rho)+\delta(N+2)-N \rho\right] .
$$

and $C S_{O L}=\left(N k_{O L}\right)^{2} / 2$ is the equilibrium level of consumer surplus. It is then easily shown that the following result holds:

\footnotetext{
${ }^{3}$ In the remainder of the paper we omit the explicit indication of the time argument for the sake of brevity.

${ }^{4}$ The proof is omitted as it replicates an analogous proof carried out by Reynolds (1987) in the duopoly case.
} 
Proposition 3.2. At the open-loop steady state equilibrium, provided $A>$ $q(\delta+\rho)$ :

- $k_{O L}, \pi_{O L}, S W_{O L}>0$ for any finite value of $N$;

- $k_{O L}$ and $\pi_{O L}$ are monotonically decreasing in $N$, while $S W_{O L}$ is monotonically increasing in $N$;

- $\lim _{N \rightarrow \infty} k_{O L}=\lim _{N \rightarrow \infty} \pi_{O L}=0$.

That is, the profit incentive measured by steady state open-loop profits attracts infinitely many firms to the market, and this is also socially efficient. Accordingly, the limit properties of the open-loop solution fully fit the acquired wisdom we are accustomed with from, say, the standard static oligopoly literature (see, e.g., Novshek, 1980). We are about to see that this is not the case when feedback effects are duly accounted for.

\section{The feedback Nash equilibrium}

Firms move simultaneously at every instant, generating a Nash equilibrium under feedback information. Firm $i$ 's Bellman equation is:

$$
\rho V_{i}(\mathbf{k})=\max _{I_{i}}\left[\pi_{i}+\frac{\partial V_{i}(\mathbf{k})}{\partial k_{i}} \dot{k}_{i}+\sum_{j \neq i} \frac{\partial V_{i}(\mathbf{k})}{\partial k_{j}} \dot{k}_{j}\right]
$$

where $V_{i}(\mathbf{k})$ is firm $i$ 's optimal value function that depends on the vector of states $\mathbf{k}=\left(k_{1}, k_{2}, \ldots k_{N}\right)$. We guess the following form for $V_{i}(\mathbf{k})$ :

$$
V_{i}(\mathbf{k})=\frac{\alpha}{2} k_{i}^{2}+\beta k_{i} \sum_{j \neq i} k_{j}+\gamma k_{i}+\varepsilon \sum_{j \neq i} k_{j}+\phi,
$$


where we attach no weight to any quadratic term $k_{j}^{2}, j \neq i$, as these do not appear in the instantaneous payoff and in the set of state equations. Moreover, the choice of coefficients reflects the a priori symmetry characterising the game. That is: (i) the weights that $i$ attaches to its own quadratic and linear terms, $\alpha$ and $\gamma$, are the same for all $i$; (ii) the weights that $i$ attaches to the mixed terms and the remaining linear ones involving rivals' capacities, $\beta$ and $\varepsilon$, are the same for all $i$; (iii) the free term $\phi$ is symmetric across firms.

The solution procedure requires taking the set of first order conditions ${ }^{5}$ on the r.h.s. of (4.1), substituting their solutions into (4.1) and simplifying the latter in order to obtain a second-degree polynomial in the states.

The first order condition for firm $i$ is:

$$
q+c I_{i}=\frac{\partial V_{i}(\mathbf{k})}{\partial k_{i}}=\alpha k_{i}+\beta \sum_{j \neq i} k_{j}+\gamma
$$

yielding:

$$
I_{i}^{*}=\frac{\alpha k_{i}+\beta \sum_{j \neq i} k_{j}+\gamma-q}{c} .
$$

Now we plug all of the expressions $I_{i}^{*}$ into (4.1), and then impose symmetry across players w.r.t. states, i.e., we set $k_{i}=k_{j}=k$ for all $i, j$. This amounts to saying that we focus our attention to a symmetric feedback Nash equilibrium. This yields the following:

$$
\begin{gathered}
\rho\left[\frac{\alpha}{2} k^{2}+(N-1) \beta k^{2}+(\gamma+\varepsilon(N-1)) k+\phi\right]= \\
(A-N k) k-q I^{*}-\frac{c}{2}\left(I^{*}\right)^{2}+N[(\alpha+\beta(N-1)) k+\gamma]\left(I^{*}-\delta k\right)
\end{gathered}
$$

which can be appropriately rewritten as follows:

$$
\frac{k^{2}}{2 c}\left[2 c N-\alpha^{2}+\beta(N-1)(2 c(2 \delta+\rho)-3 \beta(N-1))+\right.
$$

\footnotetext{
${ }^{5}$ Second-order conditions for concavity are satisfied by construction, in view of the linear-quadratic form of the problem at hand.
} 


$$
\begin{gathered}
\alpha(c(2 \delta+\rho)-4 \beta(N-1))]+ \\
\frac{k}{c}[(\alpha-\beta) \varepsilon-A c+\gamma(2 \beta-\alpha)-(\alpha \varepsilon+\beta(2 \gamma+(N-2) \varepsilon)) N+ \\
(\alpha+2 \beta(N-1)) q+c(\gamma+(N-1) \varepsilon)(\delta+\rho)]+ \\
\frac{(q-\gamma)(\gamma+2(N-1) \varepsilon-q)}{2 c}+\rho \phi=0
\end{gathered}
$$

This gives rise to the following system of three equations:

$$
\begin{gathered}
2 c N-\alpha^{2}-3 \beta^{2}(N-1)^{2}+2 c \beta(N-1)(2 \delta+\rho)+\alpha(c(2 \delta+\rho)-4 \beta(N-1))=0 \\
(\alpha-\beta) \varepsilon-A c+\gamma(2 \beta-\alpha)-(\alpha \varepsilon+\beta(2 \gamma+(N-2) \varepsilon)) N+ \\
(\alpha+2 \beta(N-1)) q+c(\gamma+(N-1) \varepsilon)(\delta+\rho)=0 \\
\frac{(q-\gamma)(\gamma+2(N-1) \varepsilon-q)}{2 c}+\rho \phi=0
\end{gathered}
$$

with five unknown parameters $(\alpha, \beta, \gamma, \varepsilon, \phi)$. Hence, we need two additional conditions in order to attain a closed form solution. Such conditions are to be constructed using economically plausible conditions that a priori the model must necessarily satisfy. We propose the following two. The first obtains exploiting the idea that, if $N=1$, the model ceases to be a game and becomes an optimal control model with a single agent (a monopolist). Accordingly, the optimal control and state yielded by the feedback solution must coincide with those produced by open-loop information. The second comes from the opposite direction, as the limit of optimal controls and states for $N$ tending to infinity should intuitively be the same (in particular, zero) under both feedback and open-loop information. That is, we invoke the acquired wisdom whereby the limit of a free entry process drives the individual firm's size (or quantity, which here is the same as size) to zero, irrespective of the information structure. In view of these additional constraints, the system (4.7) becomes determined. 
To begin with,

$$
\phi=\frac{(q-\gamma)(\gamma+2(N-1) \varepsilon-q)}{2 c \rho}
$$

Then, we solve the equation generated by the linear term w.r.t. $\gamma$, obtaining:

$$
\gamma=\frac{(\alpha+2(N-1) \beta) q-A c-\varepsilon(\alpha+(N-1) \beta)(N-1)+c \varepsilon(N-1)(\delta+\rho)}{\alpha+2(N-1) \beta-(\delta+\rho) c}
$$

Now we are left with the first equation in (4.7), that can be solved w.r.t. $\alpha$ :

$$
\alpha=\frac{c(2 \delta+\rho)-4(N-1) \beta \pm \sqrt{4 \beta^{2}(N-1)^{2}+c\left(8 N+c(2 \delta+\rho)^{2}\right)}}{2}
$$

Stability requires us to pick the negative root in (4.10).

At this point, we proceed to construct a condition based on the monopoly solution. The open-loop equilibrium capacity under monopoly is: ${ }^{6}$

$$
k_{M}=\frac{A-q(\delta+\rho)}{2+c(\delta+\rho) \delta}
$$

Imposing stationarity on (2.1) and solving it w.r.t. $k$, we obtain an expression $k(\beta, \varepsilon)$ that must be equal to $k_{M}$ in $N=1$. To this purpose, we solve $k(\beta, \varepsilon)-k_{M}=0$ obtaining $\varepsilon(\beta)$, with

$$
\left.\varepsilon(\beta)\right|_{N=1}=\frac{(q(\delta+\rho)-A)\left(c(2+\beta \rho)+\beta \sqrt{c\left(8+c(2 \delta+\rho)^{2}\right)}\right)}{(2+c(\delta+\rho) \delta)\left(c \rho+\sqrt{c\left(8+c(2 \delta+\rho)^{2}\right)}\right)}
$$

Finally, we are left with the issue of determining $\beta$. This problem can be

\footnotetext{
${ }^{6}$ See above, expression (3.6).
} 
tackled by solving: ${ }^{7}$

$$
\begin{gathered}
\lim _{N \rightarrow \infty} k(\beta, \varepsilon(\beta))=0 \Rightarrow \\
\beta=-\frac{2 c}{c \rho+\sqrt{c\left(8+c(2 \delta+\rho)^{2}\right)}}
\end{gathered}
$$

Optimal parameters can now be plugged recursively into one another as well as in the expression of $k(\beta, \varepsilon)$ to obtain the feedback equilibrium capacity level $k_{F}$ :

$$
k_{F}=\frac{4 c[A-q(\delta+\rho)](2 \Omega+c \rho)}{(2 \Theta+c \rho)[4 \Theta \Omega-c(4(N-1)+\rho(2(\Omega-\Theta)+c \rho))]}
$$

where

$$
\begin{gathered}
\Omega \equiv \frac{1}{2} \sqrt{c\left(c(2 \delta+\rho)^{2}+8\right)} \\
\Theta \equiv \frac{1}{2} \sqrt{c\left(c(2 \delta+\rho)^{2}+8 N+\frac{16 c(N-1)^{2}}{(2 \Omega+c \rho)^{2}}\right)}
\end{gathered}
$$

The corresponding equilibrium investment is $I_{F}=\delta k_{F}$. Also in this case, the pair $\left(k_{F}, I_{F}\right)$ is a saddle point equilibrium.

It is then easily checked that the following holds:

$$
\begin{gathered}
\lim _{N \rightarrow \infty} k_{F}=\lim _{N \rightarrow \infty} k_{O L}=0 \\
\left.k_{F}\right|_{N=1}=\left.k_{O L}\right|_{N=1}=\frac{A-q(\delta+\rho)}{2+c \delta(\delta+\rho)}
\end{gathered}
$$

and we can adopt plausible values for exogenous parameters, e.g., $A=10$, $q=c=1, \rho=1 / 20$ and $\delta=2 \rho$ to plot relevant magnitudes and assess their behaviour as market structure (measured by the number of firms $N$ ) varies:

\footnotetext{
${ }^{7}$ Here it is worth observing that since $\beta<0$, the game exhibits what Figuières (2009) defines as instantaneous gross payoff substitutability, in analogy with the concept of strategic substitutability identified by Bulow et al. (1985) in the static Cournot game. See also Long (2010, pp. 141-47).
} 


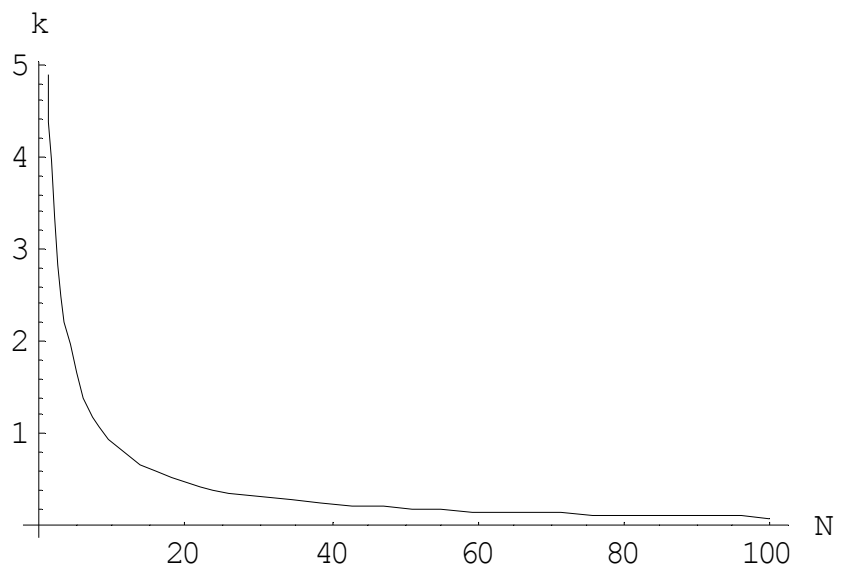

Figure 1: The individually optimal capacity at the feedback equilibrium as a function of market structure

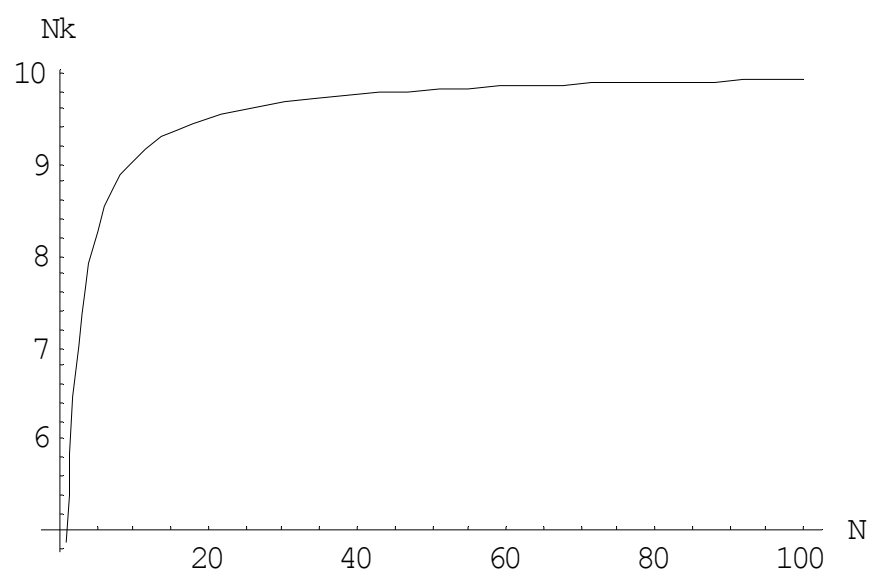

Figure 2: The capacity endowment of the industry at the feedback equilibrium as a function of market structure 


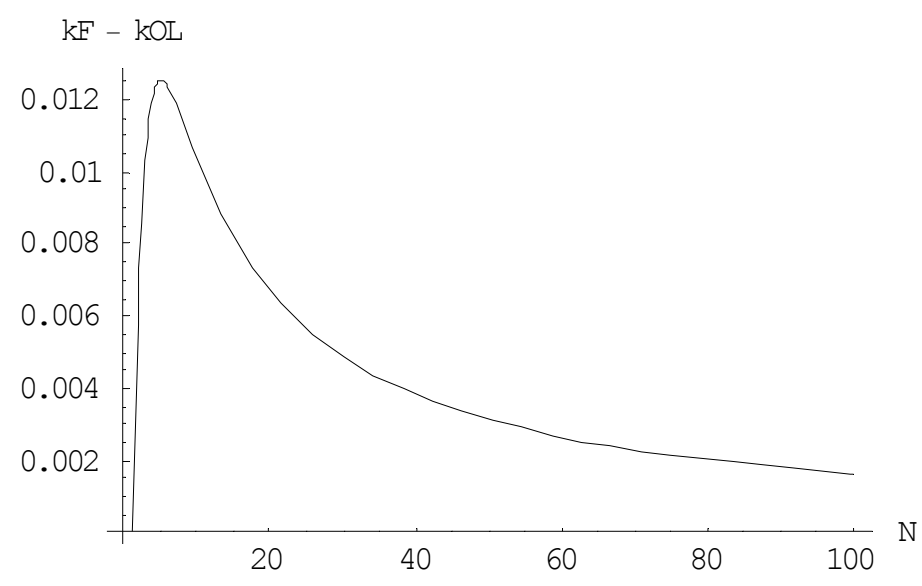

Figure 3: Individual feedback vs open-loop equilibrium capacity as a function of market structure

That is, for all finite number of firms $N \geq 2, k_{F}>k_{O L}$ and consequently $I_{F}>I_{O L}$. Consequently, $\pi_{O L}>\pi_{F}$ holds as well. However, the incentive to preempt (or equivalently, the intensified degree of strategic interaction) created by the presence of feedback information causes the feedback equilibrium profits to drop to zero in correspondence of a finite number of firms. In particular, with the numerical values we are using in this example, this happens at $N=48.79$. This, in view of the obvious integer constraint concerning $N$, implies that the maximum number of firms that may survive in the long run under feedback information is $N=48$. Checking the sign of $\partial S W_{F} / \partial N$ at $N=48$, we see that social welfare is increasing (because consumer surplus is, as any additional firm would bring about an increase in output and a decrease in price). This translates into: 
Proposition 4.1. At the feedback equilibrium, the population of firms surviving with zero profits is smaller than socially optimal.

This is possibly the most striking difference between the industry performance observed at the (subgame perfect) feedback equilibrium and that emerging from the open-loop game, and it must be entirely traced back to the excess investment in capacity driven by feedback effects intensifying strategic interaction among firms.

\section{Extensions: product differentiation, envi- ronmental externalities and Pigouvian tax- ation}

Now we can extend the model to illustrate the potential applications of our solution method. To begin with, we modify the demand function to allow for product differentiation. Each firm $i$ supplies a single variety whose inverse instantaneous demand function is

$$
p_{i}=A-k_{i}-s \sum_{j \neq i} k_{j}
$$

where the constant parameter $s \in[-1,1]$ measures the degree of complementarity (when it is negative) or substitutability (when it is positive) between any two varieties, while if $s=0$ each firm is a pure monopolist in a separate market (see, e.g., Singh and Vives, 1984).

Then, we pose that production involves a negative environmental externality (pollution) $\sigma$, evolving over time according to the state equation

$$
\dot{\sigma}=w \sum_{j=1}^{N} k_{i}-v \sum_{j=1}^{N} x_{i}-\eta \sigma
$$


where $w, v$ and $\eta$ are positive constants, and $x_{i}$ is the instantaneous R\&D effort carried out by firm $i$ to reduce pollutants. The instantaneous individual cost of green $\mathrm{R} \& \mathrm{D}$ is $C_{i}\left(x_{i}\right)=z x_{i}^{2}, z>0$. In order to provide firms with the appropriate incentives in this direction, a regulator has to impose on them at every instant a Pigouvian tax $T$ proportional to the current stock of pollution, whereby $T=\tau \sigma{ }^{8}$ For simplicity, we assume $\tau$ to be constant over time.

As a result, the instantaneous profit function of firm $i$ writes

$$
\pi_{i}=\left(A-k_{i}-s \sum_{j \neq i} k_{j}\right) k_{i}-q I_{i}-\frac{c I_{i}^{2}}{2}-z x_{i}^{2}-\tau \sigma .
$$

The firm chooses $I_{i}$ and $x_{i}$ so as to maximise the discounted profit flow

$$
\Pi_{i}=\int_{0}^{\infty} \pi_{i} e^{-\rho t} d t
$$

s.t. the set of dynamic constraints (2.1), the dynamics of pollution (5.2) and initial conditions $k_{i}(0)=k_{i 0}>0$. The discount rate $\rho>0$ is constant and common to all firms.

\subsection{Open-loop information}

Here we briefly deal with the open-loop version of the game. The Hamiltonian of firm $i$ is

$$
\mathcal{H}_{i}=\pi_{i}+\lambda_{i i} \dot{k}_{i}+\sum_{j \neq i} \lambda_{i j} \dot{k}_{j}+\varpi \dot{\sigma}
$$

where $\varpi$ is the costate variable associated with $\sigma$. The necessary conditions are:

$$
\frac{\partial \mathcal{H}_{i}}{\partial I_{i}}=-c I_{i}-q-\lambda_{i i}=0
$$

\footnotetext{
${ }^{8} \mathrm{~A}$ thorough account of the related debate is outside the scope of the present paper. See, e.g., Downing and White (1986), Milliman and Prince (1989), Benchekroun and Long (1998) and Poyago-Theotoky (2007).
} 


$$
\begin{gathered}
\frac{\partial \mathcal{H}_{i}}{\partial x_{i}}=-2 z x_{i}-\varpi v=0 \\
-\frac{\partial \mathcal{H}_{i}}{\partial k_{i}}=\dot{\lambda}_{i i}-\rho \lambda_{i i} \Leftrightarrow \\
\dot{\lambda}_{i i}=\lambda_{i i}(\delta+\rho)-A+2 k_{i}+s(N-1) k_{j}-\varpi w \\
-\frac{\partial \mathcal{H}_{i}}{\partial \sigma}=\dot{\varpi}-\rho \varpi \Leftrightarrow \dot{\varpi}=\varpi(\eta+\rho)+\tau
\end{gathered}
$$

It is worth stressing that the presence of the Pigouvian tax rate $\tau$ in the latter expression forces firms to account for the environmental implications of their primary activity. In absence of such regulation, they would simply solve equation (5.9) by setting $\varpi=0$ at all times, thereby disregarding pollution altogether, and consequently investing nothing at all to abate pollution.

From (5.7), we have

$$
\varpi=-\frac{2 z x_{i}}{v} \text { and } \dot{x}_{i}=-\frac{\dot{\varpi} v}{2 z}
$$

and, imposing symmetry across controls and states and proceeding along much the same lines as in section 3, we obtain the following system of control equations:

$$
\begin{gathered}
\dot{x}_{i}=(\eta+\rho) x-\frac{\tau v}{2 z} \\
\dot{I}=\frac{2 w z x+v[(\delta+\rho)(c I+q)-A+k(2+s(N-1))]}{c v}
\end{gathered}
$$

Then, imposing stationarity on the state-control system, we get the coordinates of the unique steady state open-loop solution:

$$
\begin{aligned}
k_{O L} & =\frac{[A-q(\delta+\rho)](\eta+\rho)-\tau w}{(\eta+\rho)[2+s(N-1)+c \delta(\delta+\rho)]} ; I_{O L}=\delta k_{O L} ; x_{O L}=\frac{\tau v}{2 z(\eta+\rho)} \\
\sigma_{O L} & =\frac{N\left[2 z((\eta+\rho)(A-q(\delta+\rho))-\tau w) w-\tau v^{2}(2+s(N-1)+c \delta(\delta+\rho))\right]}{2 z \eta(\eta+\rho)[2+s(N-1)+c \delta(\delta+\rho)]}
\end{aligned}
$$


with

$$
\sigma_{O L} \geq 0 \forall \tau \in\left[0, \frac{2 w z(\eta+\rho)(A-q(\delta+\rho))}{[2+s(N-1)+c \delta(\delta+\rho)] v^{2}+2 z w^{2}}\right]
$$

Moreover,

$$
\lim _{N \rightarrow \infty} k_{O L}=0 ; k_{M}=\frac{(\eta+\rho)(A-q(\delta+\rho))-\tau w}{(\eta+\rho)(2+c(\delta+\rho) \delta)} .
$$

\subsection{Feedback information}

We now consider the solution under feedback information. Firm $i$ 's Bellman equation is now:

$$
\rho V_{i}(\mathbf{k}, \sigma)=\max _{I_{i}, x_{i}}\left[\pi_{i}+\frac{\partial V_{i}(\mathbf{k}, \sigma)}{\partial k_{i}} \dot{k}_{i}+\sum_{j \neq i} \frac{\partial V_{i}(\mathbf{k}, \sigma)}{\partial k_{j}} \dot{k}_{j}+\frac{\partial V_{i}(\mathbf{k}, \sigma)}{\partial \sigma} \dot{\sigma}\right]
$$

where we guess the following form for $V_{i}(\mathbf{k}, \sigma)$ :

$$
V_{i}(\mathbf{k}, \sigma)=\frac{\alpha}{2} k_{i}^{2}+\beta k_{i} \sum_{j \neq i} k_{j}+\gamma k_{i}+\varepsilon \sum_{j \neq i} k_{j}+\xi \sigma+\phi,
$$

since the problem is linear in $\sigma$. The first order conditions for firm $i$ are:

$$
\begin{gathered}
q+c I_{i}=\frac{\partial V_{i}(\mathbf{k}, \sigma)}{\partial k_{i}}=\alpha k_{i}+\beta \sum_{j \neq i} k_{j}+\gamma \\
\xi v+2 z x_{i}=0
\end{gathered}
$$

yielding:

$$
\begin{gathered}
I_{i}^{*}=\frac{\alpha k_{i}+\beta \sum_{j \neq i} k_{j}+\gamma-q}{x_{i}^{*}=-\frac{\xi v}{2 z} .}
\end{gathered}
$$

Now we substitute the set of expressions $\left\{I_{i}^{*}, x_{i}^{*}\right\}$ into (5.16), and then impose symmetry across players w.r.t. states. This gives rise to the following system of four equations:

$$
k[(\eta+\rho)(c(\delta+\rho)(\gamma+\varepsilon(N-1))-A c-
$$




$$
\begin{gathered}
\beta(N-1)(2 \gamma+\varepsilon(N-1)-2 q)+\alpha(\gamma+\varepsilon(N-1)-q))-N c \tau w]=0 \\
k^{2}[c(2(1+s(N-1))+(2 \delta+\rho)(\alpha+2 \beta(N-1)))- \\
(\alpha+\beta(N-1))(\alpha+3 \beta(N-1))=0 \\
{[(\eta+r) \xi+\tau] \sigma=0} \\
\rho \phi-\left[\frac{(2 N-1) \tau^{2} v^{2}}{z(\eta+\rho)^{2}}-\frac{(q-\gamma)(\gamma+2(N-1) \varepsilon-q)}{2 c}\right]=0
\end{gathered}
$$

with six unknown parameters $(\alpha, \beta, \gamma, \varepsilon, \xi, \phi)$. As in section 4 , we will use the above system to determine four parameters and then resort to the limit properties of the steady state equilibrium to determine the remaining two.

From the last two equations we obtain

$$
\xi=-\frac{\tau}{\eta+\rho}
$$

and

$$
\phi=\frac{(2 N-1) \tau^{2} v^{2}}{z(\eta+\rho)^{2} \rho}+\frac{(q-\gamma)(\gamma+2(N-1) \varepsilon-q)}{2 c \rho}
$$

from which it appears immediately that, should $\tau=0$, the coefficient $\phi$ would indeed coincide with (4.8). Then, we solve the remaining two equations w.r.t. $\gamma$ and $\alpha$, obtaining:

$\gamma=\frac{(\alpha+2(N-1) \beta) q-A c-\varepsilon(\alpha+(N-1) \beta)(N-1)+c \varepsilon(N-1)(\delta+\rho)+c N \tau}{\alpha+2(N-1) \beta-(\delta+\rho) c}$

which coincides with (4.9) if $\tau=0$, and

$$
\alpha=\frac{c(2 \delta+\rho)-4(N-1) \beta-\sqrt{4 \beta^{2}(N-1)^{2}+c\left(8 N+c(2 \delta+\rho)^{2}\right)}}{2}
$$

which is exactly the same as in section 4 . 
Now we impose stationarity on (2.1) and solve it w.r.t. $k$, to obtain an expression $k(\beta, \varepsilon)$ that must be equal to $k_{M}$ in $N=1$, as well as

$$
\lim _{N \rightarrow \infty} k(\beta, \varepsilon)=\lim _{N \rightarrow \infty} k_{O L}=0
$$

whereby we get

$$
\left.\varepsilon(\beta)\right|_{N=1}=\frac{\beta \sqrt{c\left(8+c(2 \delta+\rho)^{2}\right)}[\tau w-(\eta+\rho)(A-(\delta+\rho) q)]+\Xi}{(\eta+\rho)(2+c(\delta+\rho) \delta)\left(c \rho+\sqrt{c\left(8+c(2 \delta+\rho)^{2}\right)}\right)}
$$

where

$$
\begin{aligned}
\Xi \equiv & c[\tau w(\beta \rho-2(2-s))+(\eta+\rho)(\delta+\rho)(\beta \rho+2 s) q]- \\
& A c(\eta+\rho)(\beta \rho+2 s)
\end{aligned}
$$

and finally

$$
\beta=\frac{2 c[s(\eta+\rho)(A-(\delta+\rho) q)+(2-s+c(\delta+\rho) \delta) \tau w]}{\left(c \rho+\sqrt{c\left(8+c(2 \delta+\rho)^{2}\right)}\right)[(\eta+\rho)(A-(\delta+\rho) q)-\tau w]} .
$$

It is relatively quick to verify that, when the goods are perfect substitutes and there is no taxation, i.e. $s=1$ and $\tau=0$, the coefficients $\varepsilon(\beta)$ and $\beta$ coincide with (4.12) and (4.14), respectively.

A comparative assessment of open-loop and feedback outcomes might seem cumbersome, in view of the lengthy expressions of the coefficients of the value function. In fact, a key result is very easily singled out:

Proposition 5.1. Given $\tau$, at the feedback equilibrium the firms' green $R \mathscr{E} D$ efforts are the same as under open-loop information. However, the steady state amount of externality is lower. 
Proof To prove this result, it is sufficient to observe that, using (5.21), one gets

$$
x^{*}=x_{O L}=\frac{\tau v}{2 z(\eta+\rho)} .
$$

That is, for any exogenously given tax rate, the intensity of $R \& D$ is unaffected by the structure of information, the reason being the linearity of the Bellman equation w.r.t. $\sigma$. Yet, as we already know from the foregoing discussion, feedback information brings about a reduction in industry output (either because of a contraction in the individual firm's output for a given industry structure, or because of an increase in concentration, or both). As a consequence, the resulting amount of pollution affecting the industry at the feedback equilibrium is lower than that associated with the open-loop solution purely because of the output reduction.

\section{Concluding remarks}

Here we have proposed a fully analytical and relatively compact solution to the feedback problem faced by a population of oligopolistic firms involved in a differential game with costly capacity adjustment à la Solow-Swan. We have shown that the limit properties of the feedback equilibrium drastically differ from those characterising the open-loop one, in particular because the maximum number of firms that may survive in the long run is infinitely high under open-loop information while it is finite under feedback information.

Alongside with the basic model, we have also illustrated its extension to a case where there exist environmental externalities increasing in the industry output. Applications of our solution method for the feedback equilibrium can of course be envisaged in several other directions, such as the analysis of horizontal mergers, corporate taxation, intraindustry trade and foreign 
direct investments. This task is left for future research.

\section{References}

Benchekroun, H. and N.V. Long (1998). Efficiency inducing taxation for polluting oligopolists. Journal of Public Economics, 70, 325-342.

Bulow, J., J. Geanakoplos and P. Klemperer (1985). Multimarket oligopoly: strategic substitutes and complements. Journal of Political Economy, 93, 488-511.

Calzolari, G. and L. Lambertini (2006). Tariffs vs quotas in a trade model with capital accumulation. Review of International Economics, 14, 632-644.

Calzolari, G. and L. Lambertini (2007). Export restraints in a model of trade with capital accumulation. Journal of Economic Dynamics and Control, 31, 3822-842.

Cellini, R., and L. Lambertini (1998). A dynamic model of differentiated oligopoly with capital accumulation. Journal of Economic Theory, 83, 145-155.

Cellini, R. and L. Lambertini (2008). Weak and strong time consistency in a differential oligopoly game with capital accumulation. Journal of Optimization Theory and Applications, 138, 17-26.

Dockner, E.J. (1992). A dynamic theory of conjectural variations. Journal of Industrial Economics, 40, 377-395.

Downing, P.B. and L.J. White (1986). Innovation in pollution control. Journal of Environmental Economics and Management, 8, 225-71. 
Driskill, R. and S. McCafferty (1989). Dynamic duopoly with adjustment costs: a differential game approach. Journal of Economic Theory, 49, 324-338.

Fershtman, C. and E. Muller (1984). Capital accumulation games of infinite duration. Journal of Economic Theory, 33, 322-339.

Figuières, C. (2009). Markov interactions in a class of dynamic games. Theory and Decision, 66, 39-68.

Jun, B. and X. Vives (2004). Strategic incentives in dynamic duopoly. Journal of Economic Theory, 116, 249-281.

Long, N.V. (2010). A Survey of Dynamic Games in Economics. World Scientific, Singapore.

Milliman, S.R. and R. Prince (1989). Firm incentives to promote technological change in pollution control. Journal of Environmental Economics and Management, 17, 247-65.

Novshek, W. (1980). Cournot equilibrium with free entry. Review of Economic Studies, 47, 473-486.

Poyago-Theotoky, J.A. (2007). The organization of R\&D and environmental policy. Journal of Economic Behavior and Organization, 62, 63-75.

Ramsey, F.P. (1928). A mathematical theory of saving. Economic Journal, 38, 543-549.

Reynolds, S. (1987). Preemption and commitment in an infinite horizon model. International Economic Review, 28, 69-88.

Reynolds, S. (1991). Dynamic oligopoly with capacity adjustment costs. Journal of Economic Dynamics and Control, 15, 491-514. 
Singh, N. and X. Vives (1984). Price and quantity competition in differentiated duopoly. RAND Journal of Economics, 15, 546-554.

Solow, R. (1956). A contribution to the theory of economic growth. Quarterly Journal of Economics, 70, 65-94.

Swan, T.W. (1956). Economic growth and capital accumulation. Economic Record, 32, 334-361. 


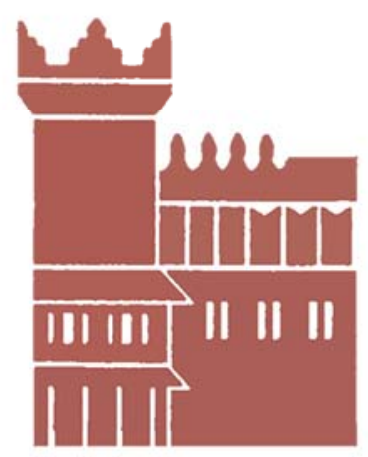

Alma Mater Studiorum - Università di Bologna DEPARTMENT OF ECONOMICS

Strada Maggiore 45

40125 Bologna - Italy

Tel. +39051 2092604

Fax +390512092664

http://www.dse.unibo.it 